\section{Identification of genes associated with stroke risk}

Two single-nucleotide polymorphisms (SNPs) associated with an increased susceptibility to stroke have been identified on chromosome 12 (locus p13). These SNPs are located in close proximity to NINJ2, a gene that encodes the adhesion molecule ninjurin 2, which is induced by nerve injury and promotes neurite regrowth.

Genomewide association studies, in which complete sets of DNA or genomes of large and diverse populations are scanned for disease markers, have identified genetic variants implicated in diabetes and coronary artery disease. However, studies of genetic variation as an underlying factor in stroke have produced inconclusive results. "Stroke has a high case-fatality, so genomewide association studies on prevalent stroke cases and controls might miss genes associated with the most severe strokes," says Sudha Seshadri, one of the researchers from Boston University. With access to data that had been collected in four prospective, population-based cohort studies, Seshadri and colleagues from the US and Europe studied genetic associations with incident stroke in more than 19,000 people. Their analysis identified two SNPs that were consistently associated with stroke. Most of the study participants were Caucasian, but one of the SNPs was also found in a replication cohort of African Americans with incident stroke.

The proximity of the SNPs to NINJ2, which is thought to promote repair of damaged nerves, indicates that they might be involved in the response to brain injury, including ischemia-induced injury. However, much more work is required to verify whether NINJ2 and its variants do indeed have a role in response to nerve injury. In collaboration with other groups that have genomewide association data, Seshadri and colleagues plan to perform a meta-analysis to identify other genetic variants that could be associated

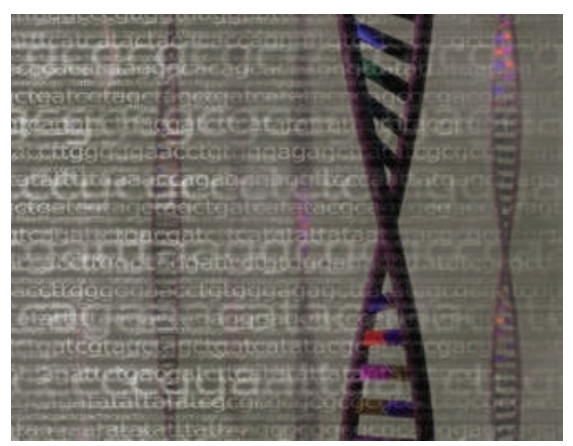

with stroke. They have also initiated sequencing studies to identify and characterize the causal SNPs. "Identifying novel loci expands our understanding of pathophysiology and may lead to new preventive and treatment strategies in 5-10 years. It could also help to refine risk prediction," says Seshadri, adding that "this needs to be studied in more detail".

Sharmini Rajanayagam

Original article Ikram, M. A. et al. Genomewide association studies of stroke. N. Engl. J. Med. 360, 1718-1728 (2009). 